\title{
Hemoperfusion with HA380 in acute type $A$ aortic dissection patients undergoing aortic arch operation (HPAO): a randomized, controlled, double-blind clinical trial
}

Jing Yang, Dong Ji, Yue-Qian Zhu, Yun Ren, Xun Zhang, Hong-Yu Dai, Xu Sun, Yi Zhou, Zhi-Yuan Chen, Qing-Guo Li and Hao Yao* (D)

\begin{abstract}
Background: Cardiopulmonary bypass (CPB) is an important cause of significant systemic inflammatory response syndrome (SIRS) in the surgical treatment of acute type A aortic dissection (ATAAD). In patients with arch vessel involvement, extensive surgical repairs often necessitate prolonged use of CPB and results in extensive inflammatory responses. Cytokines and chemokines released during CPB contribute to the progression of SIRS, increase perioperative complications, and negatively impact surgical outcomes. A cytokine adsorber (HA380) is expected to reduce the level of cytokines during CPB, which may decrease both intraoperative and postoperative inflammation. The purpose of this study is to investigate if HA380 is able to reduce the levels of inflammatory cytokines and decrease perioperative complications in ATAAD patients undergoing CPB and deep hypothermic circulatory arrest (DHCA).
\end{abstract}

Methods: This study is a single-center, randomized, controlled, double-blind clinical trial. The study aims to recruit 88 patients with ATAAD and aortic arch involvement who will undergo CPB and DHCA to repair the dissected aorta. Patients will be randomized equally into the CPB/DHCA only group (control group) and the CPB/DHCA + HA380 hemoperfusion group (intervention group), with 44 patients each. Patients in the control group will undergo CPB and DHCA only, while patients in the intervention group will undergo continuous hemoperfusion with HA380, in addition to CPB and DHCA. The primary outcome is a composite of major perioperative complications. The secondary outcomes include related inflammatory markers, coagulation parameters, and minor perioperative complications. To comprehensively evaluate the effect of hemoperfusion on the perioperative outcomes, we will also determine if there are differences in perioperative all-cause mortality, length of ICU stay, and total hospitalization costs.

* Correspondence: liqg@njmu.edu.cn; yaohao@njmu.edu.cn Cardiovascular Center, The Second Affiliated Hospital of Nanjing Medical University, Nanjing 210011, Jiangsu Province, China

C C The Author(s). 2020 Open Access This article is licensed under a Creative Commons Attribution 4.0 International License, which permits use, sharing, adaptation, distribution and reproduction in any medium or format, as long as you give appropriate credit to the original author(s) and the source, provide a link to the Creative Commons licence, and indicate if changes were made. The images or other third party material in this article are included in the article's Creative Commons licence, unless indicated otherwise in a credit line to the material. If material is not included in the article's Creative Commons licence and your intended use is not permitted by statutory regulation or exceeds the permitted use, you will need to obtain permission directly from the copyright holder. To view a copy of this licence, visit http://creativecommons.org/licenses/by/4.0/ The Creative Commons Public Domain Dedication waiver (http://creativecommons.org/publicdomain/zero/1.0/) applies to the data made available in this article, unless otherwise stated in a credit line to the data. 
(Continued from previous page)

Discussion: In the current trial, hemoperfusion will be applied in patients undergoing CPB and DHCA for repair of the aorta involving the aortic arch. This trial aims to test the safety and efficacy of our hemoperfusion device (HA380) in such settings. Upon completion of the trial, we will determine if HA380 is effective in reducing perioperative proinflammatory cytokine levels. Further, we will also verify if reduction in the proinflammatory cytokine levels, if present, translates to improvement in patient outcomes.

Trial registration: ClinicalTrials.gov NCT04007484. Registered on 1 July 2019 (retrospectively registered).

Keywords: HA380, Acute type A aortic dissection, Cardiopulmonary bypass, Deep hypothermic circulatory arrest, Inflammatory factors, Coagulation indicators

\section{Background}

Cardiovascular disease is the leading cause of death worldwide [1]. In the last couple of decades, the advancement of cardiac surgery has enabled invasive management and improved overall outcomes in patients with heart and great vessel diseases. Cardiopulmonary bypass $(\mathrm{CPB})$ is an indispensable technique used in open-heart surgery that ensures perfusion of vital organs with a stopped heart. While essential, CPB can induce the massive release of inflammatory cytokines, causing overwhelmed systemic inflammation reactions that may increase perioperative mortality.

It is estimated that the incidence of systemic inflammatory response syndrome (SIRS) in patients undergoing $\mathrm{CPB}$ can reach as high as $40 \%$ to $50 \%$ [2, 3]. During $\mathrm{CPB}$, contact of blood with artificial materials activates monocytes and macrophages, both of which release inflammatory cytokines and induce inflammation [4]. In patients who developed SIRS after CPB, white blood cell count and multiple inflammatory markers such as interleukin (IL)-6, IL-8, and tumor necrosis factor- $\alpha$ (TNF- $\alpha$ ) were all significantly increased [5-7]. In addition, the severity of SIRS, marked by inflammatory cytokines, after cardiac surgery directly correlates to the perioperative complications and prognosis [8-10].

Acute type A aortic dissection (ATAAD) is a cardiovascular emergency that requires immediate open surgical treatment. In the surgical treatment of ATAAD, the extensive repair necessitates prolonged use of $\mathrm{CPB}$, which exacerbates inflammation and negatively impact surgical outcomes. Because the levels of inflammatory markers influence the severity of SIRS and prognosis, measures to reduce inflammation have always been the center of focus in open-heart surgery.

Hemoperfusion is a technique widely used in toxicology for the removal of toxins. It works by removing cytokines that cannot be filtered using existing blood purification techniques [11]. Recent studies suggested that hemoperfusion is also able to absorb inflammatory mediators produced during $\mathrm{CPB}$ and produce a longlasting anti-inflammatory effect [12]. However, the role of hemoperfusion in the settings of ATAAD patients undergoing $\mathrm{CPB}$ and deep hypothermic circulatory arrest (DHCA) is currently unknown. In the present study, we would like to determine if hemoperfusion is able to reduce inflammatory mediators and reduce the perioperative complications in ATAAD patients undergoing $\mathrm{CPB}$ and DHCA.

\section{Objective and hypothesis}

This study is a single-center, randomized, controlled, double-blind clinical trial. Subjects undergoing DHCA/ $\mathrm{CPB}$ will be allocated to the hemoperfusion group and control group. The main purpose of this study is to determine if the levels of pro-inflammatory cytokines and anti-inflammatory cytokines differ between the two study groups in the perioperative period. In addition, differences in perioperative coagulation parameters, blood product usages in the intensive care unit (ICU), length of hospital stay, postoperative complications, and allcause mortality between two groups will also be determined. We hypothesize that hemoperfusion could improve the overall prognosis in patients with acute type A aortic dissection undergoing cardiopulmonary bypass and DHCA.

\section{Methods \\ Ethics and registration}

This prospective, randomized, double-blind controlled study started recruiting patients at the Cardiovascular Center of the Second Affiliated Hospital of Nanjing Medical University in China in January 2019 and is expected to complete within 24 months (30 December 2020). The design of the trial follows the guidelines in the Helsinki Declaration and is in accordance with the guidelines of the Medical Research Involving Human Subjects Act and Good Clinical Practice (GCP). This trial has been approved by the Ethics Committee of the Second Affiliated Hospital of Nanjing Medical University (No.[2018] KY 118) and has been registered at ClinicalTrials.gov (NCT 04007484). According to the Consolidated Standards of Reporting Trials (CONSORT) statement, the study flow chart is developed, as shown in Fig. 1. 


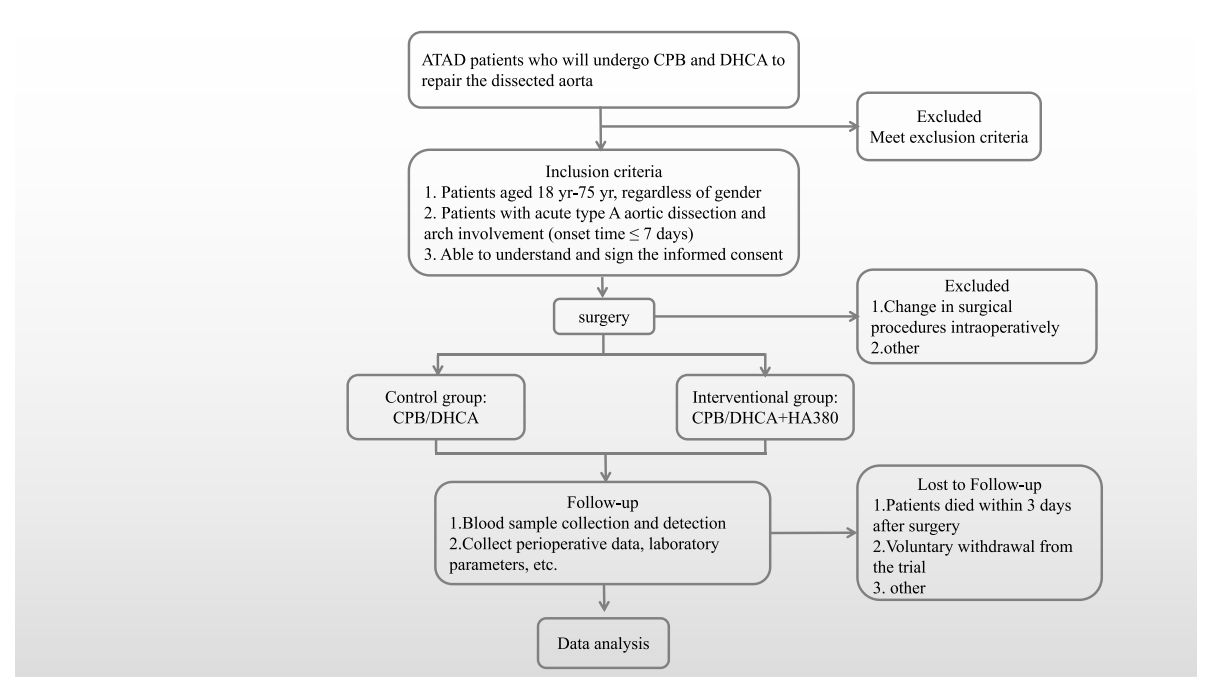

Fig. 1 Flow chart of the hemoperfusion in acute type A aortic dissection patients undergoing aortic arch operation (HPAO) trial

\section{Patient and public involvement}

Patients or the public are not involved in the design, conduct, reporting, or dissemination plans of our research.

\section{Research objects \\ Inclusion criteria}

1. Patients aged $18-75$ years, regardless of gender

2. Patients with acute type A aortic dissection and arch involvement (onset time $\leq 7$ days)

3. Able to understand and sign the informed consent

\section{Exclusion criteria}

1. Unable to understand and sign the informed consent

2. $\mathrm{BMI} \geq 40$

3. Pregnant

4. Active hemorrhage or thrombocytopenic purpura

5. Previous history of liver diseases, renal insufficiency, or cerebrovascular diseases

6. Prolonged operation time due to intraoperative accident

7. Preoperative organ malperfusion

8. Previous history of cardiac surgeries

9. Oral anticoagulant or antiplatelet drugs within 1 week of disease onset

10. Hereditary connective tissue diseases such as Marfan syndrome, Ehlers-Danlos, and Loeys-Dietz syndrome

The listed exclusion criteria are set to minimize the impact of perioperative variables, which may potentially undermine the validity of the study. The exclusion criteria are also backed up by a number of studies focusing on cardiac surgery [13-15].
Experimental intervention/treatment

Hemoperfusion is achieved through blood filtration with a hemoperfusion device named HA380 (Zhuhai Jafron Biomedical, China). The hemoperfusion device, composed of multiple parallel macroporous adsorption resin cartridges, is connected in parallel to the oxygenator and blood reservoir and perfused at a rate of $400-700 \mathrm{ml} / \mathrm{min}$ (Fig. 2). Thanks to the large medium and macroporous structure and high specific surface area, HA380 is able to adsorb superfluous inflammatory factors and excess metabolites in the blood through different forces. The outbreak of inflammatory factors is thus expected to be controlled. The aim of hemoperfusion is to decrease inflammatory mediators produced during cardiopulmonary bypass through adsorption. The ultimate goal of hemoperfusion is to decrease postoperative complications, improve postoperative recovery, and shorten the length of hospital stay. Montin et al. detailed the safety and effectiveness of HA380. General recommendations of HA380 in cardiac surgery have also been made [16].

\section{Randomization and blinding}

On the day of surgery, the perfusionists will explain to the patient's families the context and significance of our trials and obtain informed consents. All informed consents will be kept in a separate folder and preserved by the project leader. Upon anesthesia, a statistician who does not participate in the experiments will use a computer software to randomly designate a number of either 0 or 1 to the patient, with 0 for the control group and 1 for the intervention group. The expected number of patients in the control group will equal that of the intervention group. To keep the trial confidential, the results of random allocation will only be known to the statisticians and perfusionists. Other physicians including cardiac 


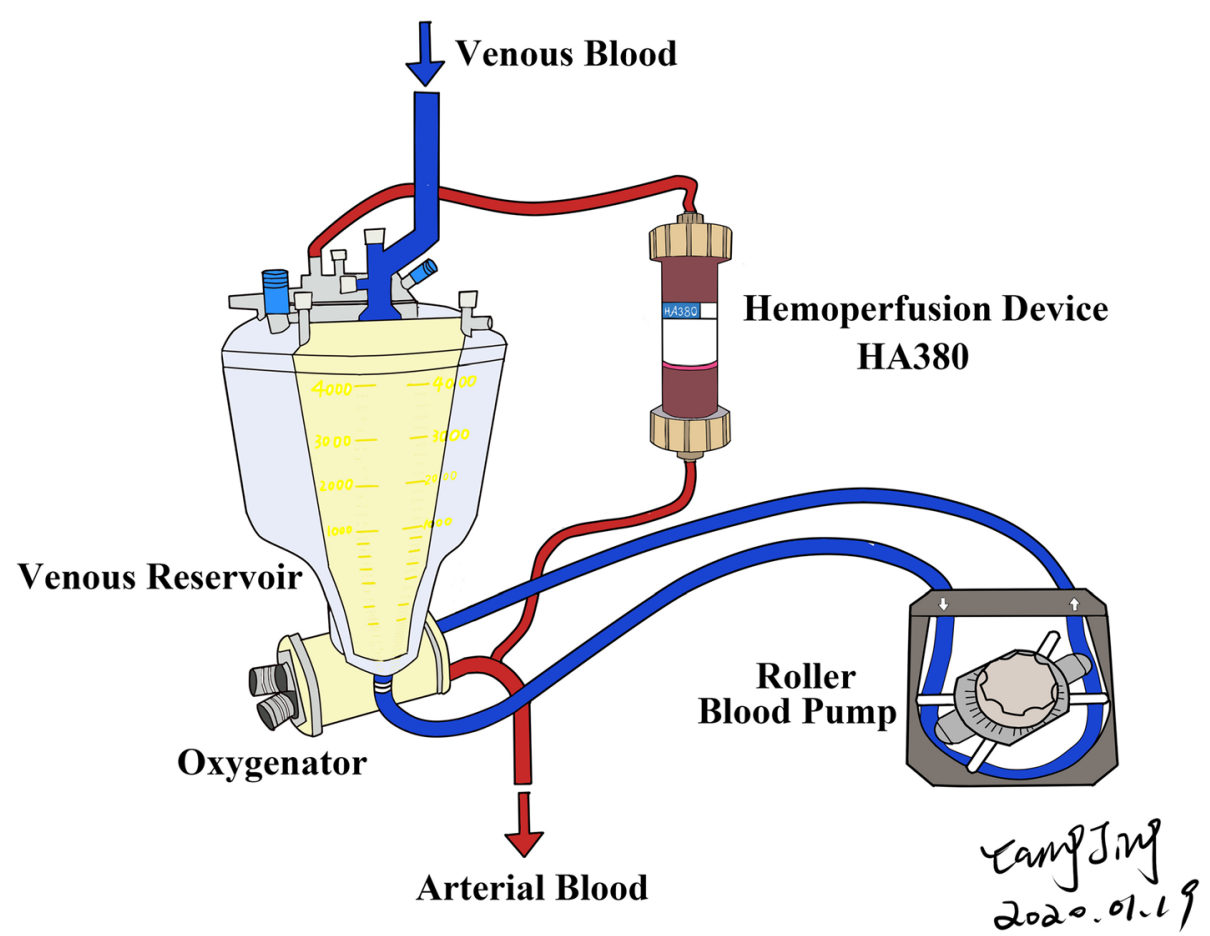

Fig. 2 HA380 hemoperfutor setup in the CPB circuit. Venous blood first enters the venous reservoir, where it subsequently flows into the oxygenator with a driving force from a roller pump. After oxygenation, the majority of the oxygenated blood goes into the arterial circulation for perfusion, roughly $700 \mathrm{ml} / \mathrm{min}$ (14-18\%) goes through the hemoperfusion device HA380 for hemofiltration. The filtered blood then returns to the venous reservoir and mixes with the venous blood. No other additional equipment is required

surgeons, anesthesiologists, intensivists, and other clinicians will be blinded to the grouping. The details of randomization will not be accessible to anyone but the statisticians and perfusionists until the end of the trial and completion of the data analysis. If exclusion criteria are met after randomization (e.g., hemi-arch replacement is performed or deep hypothermia circulatory arrest not required), the patient will be removed from the trial.

During the hospital stay, only the perfusionists are aware of the grouping of patients. First of all, the size of HA380 is small. It is often not noticeable to the surgical team. Secondly, the shape of the device is no different to conventional filters used in CPB. Therefore, it is difficult to tell if HA380 is used just by eyeballing on the CPB circuit. Third, our HA380 are specially ordered and no label is visible on the device. Thus, only the perfusionist knows if the device was used. Cardiac surgeons, intensivists, and other clinicians actively involved in the treatment of the patients are blinded to patient grouping. The detail of randomization will not be revealed until the end of the study and completion of data analysis.

\section{Data collection using case report form (CRF)}

Preoperative data including preoperative demographics (sex, age, BMI), details of presentation (onset time, symptoms such as chest pain, back pain, dyspnea, abdominal pain/abdominal distension, nausea/vomiting, coma, etc.), prior medical history (hypertension, hyperlipidemia, diabetes, coronary heart disease, kidney disease, cerebrovascular disease, smoking history, drinking history, etc.), preoperative imaging and laboratory results (such as electrocardiogram, cardiac ultrasound, computed tomography angiography, coagulation routine, liver and kidney functions, blood routine, etc.), and preoperative medications (norepinephrine, dopamine, urapidil, epinephrine, etc.) will be collected.

Intraoperative data including surgical methods, $\mathrm{CPB}$ details ( $\mathrm{CPB}$ time, aortic cross-clamp time, circulatory arrest time, unilateral cerebral perfusion time, nasopharyngeal minimum temperature, etc.), volume of intraoperative blood products (such as suspended red blood cells, fresh frozen plasma, platelet, cryoprecipitate, etc.), amount of blood loss, and urine output will also be collected.

Postoperative follow-up data including major postoperative complications (new renal failure-requiring dialysis, secondary thoracotomy, operative mortality, stroke/ cerebrovascular accident, paraplegia, acute renal injury, respiratory failure, delirium, severe liver dysfunction, myocardial infarction, etc.), general condition after operation (duration of mechanical ventilation, drainage volume within $24 \mathrm{~h}$ postoperatively, urine volume within $24 \mathrm{~h}$ postoperatively, the length of ICU stay, all-cause 
mortality, dosage of vasoactive drugs, total hospitalization cost, etc.), and volume of postoperative blood product transfusions will be collected. Utilization of invasive postoperative treatment methods such as continuous renal replacement therapy (CRRT) or extracorporeal membrane oxygenation (ECMO) will be documented as well. In general, all patients enrolled will be followed up at our center. Part of the data to be collected throughout the study is shown in Fig. 3.

It should be noted that contrast-enhanced thoracic computed tomography aortography (CTA) needs to be provided before operation to confirm the diagnosis, and the data of preoperative examinations is the closest one to the operation. Preoperative and postoperative data will be collected and recorded by team members who are blinded to surgical details and randomization. Intraoperative data will be collected by perfusionists and anesthesiologists.

\section{Anesthesia and surgical procedures}

Venous access is achieved through peripheral vein puncture in the operating room. Throughout the surgery, five-lead electrocardiogram (ECG), blood oxygen saturation $\left(\mathrm{SPO}_{2}\right)$, and heart rate (HR) should be routinely monitored with patient monitor (Philips, Holland). Noninvasive cerebral oxygen saturation $\left(\mathrm{rSO}_{2}\right)$ and $\mathrm{BIS}$ monitoring are achieved with Disposable sensor (Covidien, USA). During the operation, the value of $\mathrm{rSO}_{2}$ is maintained at no less than $80 \%$ of baseline with an absolute value greater than $50 \%$. Induction of anesthesia is achieved with etomidate 10 to $15 \mathrm{mg}$, sufentanil $0.5-1 \mu \mathrm{g} / \mathrm{kg}$, and cis-atracurium $0.15 \mathrm{mg} / \mathrm{kg}$ intravenously. Anesthesia is maintained with dexmedetomidine $0.2-0.4 \mu \mathrm{g} / \mathrm{kg} / \mathrm{h}$, sufentanil $1-3 \mu \mathrm{g} / \mathrm{kg} / \mathrm{h}$, cisatracurium $0.06-0.12 \mathrm{mg} / \mathrm{kg} / \mathrm{h}$, and sevoflurane, at a BIS between 40 and 60 .

After median thoracotomy, the supra-aortic branches are isolated. Once ACT reaches $480 \mathrm{~s}$, cardiopulmonary bypass is started. Throughout the $\mathrm{CPB}$, the flow rate is kept at 2 to $2.51 / \mathrm{min} / \mathrm{m}^{2}$, and the mean arterial pressure is maintained between 50 and $70 \mathrm{mmHg}(1 \mathrm{mmHg}=$ $0.133 \mathrm{kPa}$ ). Cooling down of the patient is subsequently initiated while ascending aorta is being opened and false lumen thrombus being removed. Subsequently, the dissected proximal aorta is replaced with or without replacement of the prosthetic aortic valve while the patient is being cooled down. To minimize cerebral ischemia, arch vessels are reconstructed with the following sequence respectively: the left carotid artery, the left subclavian artery, and the right innominate artery. After completion of repair and rewarming, the patient is weaned from CPB. Protamine is given to reverse the effect of heparin if needed. The procedure completes after hemostasis is reached and the sternum is closed.

\section{Blood sample collection and detection}

During the perioperative period, plasma inflammatory factors levels will be measured at the following time points: before operation (T0), CPB initiation (T1), lowest intraoperative temperature (T2), end of $\mathrm{CPB}$ (T3), end of the operation (T4), $24 \mathrm{~h}$ after completion of the procedure (T5), $48 \mathrm{~h}$ after completion of the procedure (T6), and $72 \mathrm{~h}$ after completion of the procedure (T7).

Parameters of coagulation including plasma-activated partial thromboplastin time (APTT), prothrombin time (PT), fibrinogen (FIB), fibrinogen degradation product (FDP), and d-dimer (DDi) levels will be measured at the following time points: before operation, $24 \mathrm{~h}$ after completion of the procedure (POD 1), $48 \mathrm{~h}$ after completion of the procedure (POD 2 ), and $72 \mathrm{~h}$ after completion of the procedure (POD 3).

Similarly, levels of coagulation factors including II, VII, IX, X, XI, and XII will be measured at the following time points: before operation, end of $\mathrm{CPB}$, end of the operation, 24 $\mathrm{h}$ after completion of the procedure (POD 1), and $48 \mathrm{~h}$ after completion of the procedure (POD 2).

Measurement of inflammatory cytokines levels: blood samples are collected through radial artery catheterization. The first $5 \mathrm{ml}$ will be discarded when sampling. Following collection, blood samples are immediately centrifuged at $3000 \mathrm{rpm}$ for $15 \mathrm{~min}$ at $4{ }^{\circ} \mathrm{C}$. The plasma yielded will be stored in the $-80^{\circ} \mathrm{C}$ refrigerator till the time of measurements. Enzyme-linked immunosorbent assay (ELISA) is used for the measurement of inflammatory cytokines.

\section{Primary outcome measures}

1. Incidence of composite major complications [time frame: up to 30 days]:

- Operative mortality

- Secondary thoracotomy

- New onset of postoperative renal failure requiring dialysis: increase in serum creatinine value $\geq 300 \%$ from baseline or an absolute serum creatinine $\geq 354 \mu \mathrm{mol} / \mathrm{l}$ with an increase $\geq$ $44 \mu \mathrm{mol} / \mathrm{l}$ from baseline (KDIGO stage 3) [17]. Any patient who received acute dialysis is put into this category. Patients who have received dialysis within 1 month prior to the surgery are not eligible for this endpoint

- Paraplegia

- Stroke/cerebrovascular accidents

- Low cardiac output syndrome: cardiac index < $2.01 \mathrm{~min}^{-1} \mathrm{~m}^{-2}$ for $>8 \mathrm{~h}$ after surgery, regardless of treatment [18]

- Reintubation

- Severe liver dysfunction: ALT level above $500 \mathrm{U} /$ l or a total bilirubin above $3.0 \mathrm{mg} / \mathrm{dl}[19,20]$

- ECMO support

- Multiple organ dysfunction syndrome (MODS) 


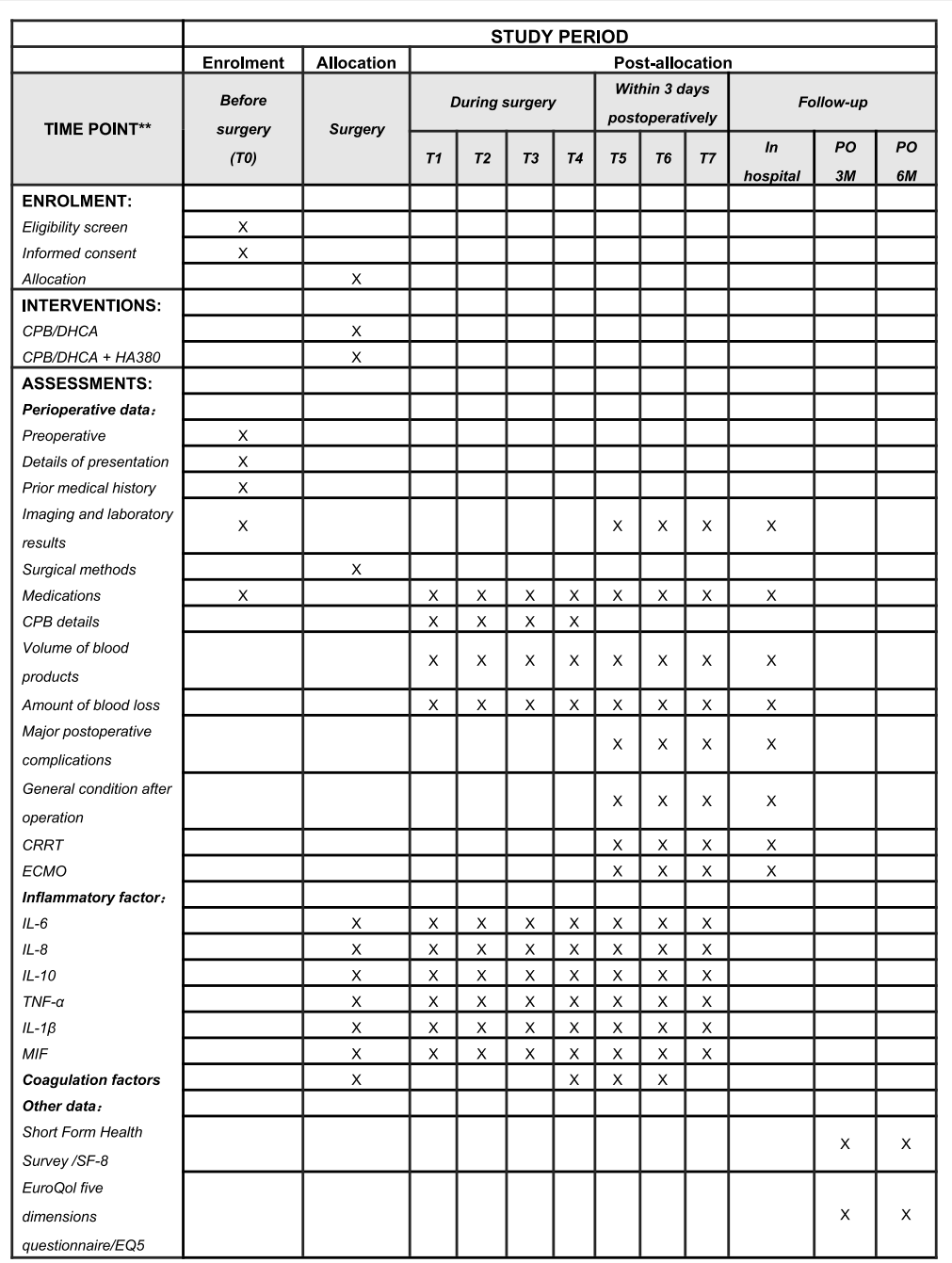

Fig. 3 Schedule of enrollment, intervention, perioperative data, and laboratory parameters at different time points on the basis of Standard Protocol Items: Recommendations for Interventional Trials (SPIRIT) Statement. T0, before operation; T1, CPB initiation; T2, lowest intraoperative temperature; $T 3$, end of $C P B ; T 4$, end of the operation; $T 5,24 \mathrm{~h}$ after completion of the procedure; $T 6,48 \mathrm{~h}$ after completion of the procedure; $T 7$, $72 \mathrm{~h}$ after completion of the procedure; CPB, cardiopulmonary bypass; DHCA, deep hypothermic circulatory arrest; CRRT, continuous renal replacement therapy; ECMO, extracorporeal membrane oxygenation; MIF, macrophage migration inhibitory factor; PO 3M, postoperative 3 months; PO 6M, postoperative 6 months

Each of these outcomes will be analyzed as a secondary endpoint.

\section{Secondary outcome measures}

1. Changes in the levels of plasma inflammatory factors such as IL- 6 , IL- 8 , IL-10, IL-1 $\beta$, and TNF- $\alpha$ [time frame: up to 3 days]

2. Changes of plasma MIF levels during the perioperative period [time frame: up to 3 days]

3. Changes of plasma CRP levels during the perioperative period [time frame: up to 3 days]

4. Changes of the plasma II, VII, IX, X, XI, and XII levels during the perioperative period [time frame: 2 days]
5. Changes of the plasma APTT, PT, FIB, FDP, and DDi levels during the perioperative period [time frame: 3 days]

6. Total drainage within the first $24 \mathrm{~h}$ of surgery [time frame: $24 \mathrm{~h}$ ]

7. Incidence of postoperative acute kidney injury [time frame: up to 30 days]: increase in serum creatinine $\geq 150 \%$ or $\geq 26.5 \mu \mathrm{mol} / \mathrm{l}$ from baseline [13]

8. Incidence of postoperative respiratory failure [time frame: up to 30 days]: uninterrupted postoperative mechanical ventilation for more than $48 \mathrm{~h} \mathrm{[10]}$

9. Incidence of postoperative delirium [time frame: up to 30 days]: we will assess delirium once a day for a total of 3 times during the first $72 \mathrm{~h}$ after ICU admission, using standard delirium assessments 
(CAM or CAM-ICU). Before the CAM-ICU will be performed, a Richmond Agitation Sedation Scale (RASS) sore should be evaluated in advance [21]

10. Incidence of postoperative liver injury [time frame: up to 30 days]: ALT level above $150 \mathrm{U} / 1$ [22]

11. Incidence of postoperative myocardial infarction [time frame: up to 30 days]: a rise and/or fall of cTn values with at least 1 value above the 99th percentile upper reference limit and at least 1 of the following:

- Symptoms of myocardial ischemia

- New ischemic ECG changes

- Development of pathological Q waves

- Imaging evidence of new loss of viable myocardium or new regional wall motion abnormality in a pattern consistent with an ischemic etiology

- Identification of a coronary thrombus by angiography or autopsy (not for types 2 or 3 MIs) [23]

\section{Other outcome measures}

1. The volume of blood transfusion within ICU [time frame: up to 30 days]

2. The need of vasoactive drugs [time frame: up to 30 days]

3. Length of ICU stay [time frame: up to 30 days]

4. Length of postoperative hospital stay [time frame: up to 30 days]

5. Prolonged postoperative extubation $(>48 \mathrm{~h})$ [time frame: up to 30 days]

6. Total hospital expenses [time frame: up to 6 months]

\section{Sample size calculation}

Primarily, we expect the incidence of composite major complications to be decreased in the HA380 hemoperfusion group (intervention group) compared with the control group. The sample size was calculated with our preliminary results from the pilot study, in which the primary outcome was defined as the incidence of composite major complications. In this study, we included 24 patients ( $n=12$ in each group), in which $8.3 \%$ ( 1 out of 12) of the patients assigned to the intervention group underwent CRRT due to severe postoperative renal failure. In contrast, 33.3\% (4 out of 12) patients had either one of the following outcomes: death, secondary thoracotomy, and renal failure requiring dialysis. Assuming a two-sided type I error probability $(\alpha)$ of $5 \%$ and type II error probability $(\beta)$ of $20 \%$, forty patients are needed in each group. Suppose $10 \%$ of patients drop out either due to changes in surgical methods or lost in follow-up, a minimum number of 44 patients in each group are needed in order to achieve statistical significance.

\section{Data management and quality control}

A safety monitoring committee composed of cardiovascular surgeons, anesthesiologists, perfusionists, and statisticians will be set up to monitor the progress and safety of the clinical study, including adverse events and mortality. All adverse events will be recorded in the case report form with severity assessed. A team member who is not involved in grouping, surgery, and postoperative management will be assigned to summarize the data. If for any reason a subject could not adhere to the study, he or she will be removed from the study groups and the sampled data will also be abandoned. On rare occasions, some of the blood samples withdrawn before emergent surgery may not meet the requirement for lab studies (hemolysis happened during blood withdrawn, blood clotted or the sample was contaminated, etc.). Should it occur, multiple imputation will be used to handle the missing data. However, if many pieces of data are missing, or vital data are missing (such as patient outcomes), the subject will be removed from the study group. Throughout the trial, only the project leader has access to the patient's data and make the final decision to terminate the trial. All information will be shared with the trial team upon completion of the study.

For quality control, all perfusionists and anesthesiologists will receive unitive training to ensure correct installation and operation of the HA380 hemoperfutor throughout the trial. The trial is overseen by the Ethics Committee of the 2nd affiliated hospital of Nanjing Medical University and will be audited every 6 months. All saved data will be kept confidentially in accordance with standard guidelines. All changes to the data first keyed in will be documented and a valid reason is required for data revision.

\section{Data analysis}

After completion of the trial, data analysis will be done by a certified statistician with SPSS20.0 (IBM, Chicago, IL, USA). Levels of inflammatory cytokines and coagulation parameters will be expressed as mean \pm standard deviation or median (interquartile range). An independent $t$ test or the Wilcoxon signed-rank test will be used to check if the data is within normal distribution before analysis. Categorical variables will be analyzed with chisquared and Fisher's exact tests.

The relative risk of primary and secondary outcomes in the hemoperfusion group and the control group will be compared using the multivariate logistic regression. Kaplan-Meier curves and log-rank analysis will be used to compare inter-group differences in primary and secondary outcomes. When comparing the changes in coagulation factor levels during the perioperative period, the effect of hemoperfusion on coagulation factors throughout the study will be assessed using repeated 
measures' analysis of variance (ANOVA) and analysis of covariance (ANCOVA). When the $p<0.05$, the result is considered statistically significant.

\section{Discussion}

During $\mathrm{CPB}$, increased levels of pro-inflammatory cytokines and decreased levels of anti-inflammatory cytokines activate the immune system $[24,25]$. The inflammation can be even more severe when deep hypothermic circulatory arrest is needed.

Most cytokines are small soluble proteins $(<40 \mathrm{kDa})$ involved in the signal transduction pathways that play key roles in the immune response [26]. The balance of pro-inflammatory and anti-inflammatory cytokines often determines the pattern of inflammation. When there is an uncontrolled release of pro-inflammatory cytokines and overactivation of the immune system, a hyperinflammatory state called cytokine storms ensues. This significantly complicates the postoperative recovery and increases the incidence of MODS and death [27-29]. We therefore set the incidence of composite major complications as the primary outcome in the instant study.

Major proinflammatory cytokines produced in CPB include IL-1, IL-6, IL-12, and TNF- $\alpha$ [24]. IL-6, a cytokine released by tissue macrophages, is a pro-inflammatory cytokine that is increased early during the inflammatory process. Its level increases significantly after initiation of $\mathrm{CPB}$ and peaks at 2-4h after the end of $\mathrm{CPB}$ [7]. Additionally, increased levels of IL- 6 increase the incidence of postoperative complications such as ARDS, myocardial ischemia, and low cardiac output syndrome [6]. Measures in decreasing the amount of IL-6 has been suggested to preserve vascular barrier integrity, reduce capillary leakage, and alleviate postoperative inflammations [30]. Consequently, we included the levels of IL-6 as one of the secondary outcomes.

Anti-inflammatory cytokines (such as IL-1RA, IL-4, and IL-10) play an equally important role in host defense [31, 32]. Among the aforementioned cytokines, IL-10 is an important anti-inflammatory cytokine that regulates the compensatory anti-inflammatory response syndrome (CARS) against SIRS [33]. However, elevated IL-10 levels can also increase the incidence of postoperative infection and sepsis [34]. Therefore, assessing the levels of IL-10 can help predict the incidence of postoperative infections.

Our study will also aim to evaluate the changes of postoperative blood coagulation function and determine the new mechanisms of postoperative organ failure. Macrophage migration inhibitory factor (MIF) is a secretory homotrimer that plays a key role in promoting the production of proinflammatory cytokines [35-37]. With a molecular weight of $12.5 \mathrm{kD}$, MIF can be effectively removed by the HA380 hemoperfusion. Removal of MIF may have a protective effect on the central nervous system and reduce the incidence of postoperative delirium. Further, free hemoglobin and myoglobin can also be readily removed by the HA380 hemoperfutor during $\mathrm{CPB}$. Because both hemoglobin and myoglobin are involved in the occurrence of cardiac surgery-associated acute kidney injury (CSA-AKI) [38], hemoperfusion may have an additional protective effect on the kidney and may contribute to the recovery of renal function after cardiac surgery.

Because the interplay between inflammation and coagulation systems seriously worsens the prognosis of patients undergoing surgery, we therefore aim to evaluate if hemoperfusion could reduce abnormal bleeding and the amount of transfusion after the aortic arch operation. Additionally, in order to evaluate the safety of HA380 hemoperfutor, we will also detect the levels of perioperative coagulation factors such as II, VII, IX, X, XI, XII, and so on.

Steroids are commonly used for the excessive inflammation in ATAAD patients with severe postoperative inflammation [39]. While effective, steroid uses are not without side effects. Hyperglycemia and increased risk of postoperative infection are common side effects seen with steroids. Another anti-inflammatory drug, ulinastatin, can cause granulocytopenia and allergies. Therefore, other emerging technologies such as hemofiltration are needed to address the limitations of medications. Though well tolerated, one limitation with the hemoperfusion technologies is the inconsistent filtration and adsorption results.

HA380 is a new hemoperfusion device [16]. In this trial, we evaluated the effect of the hemoperfusion device (HA380) on the perioperative inflammatory responses in ATAAD patients undergoing $\mathrm{CPB}$ and DHCA. The purpose of this study is to investigate if hemoperfusion with HA380 is sufficient to reduce perioperative proinflammatory cytokine levels and reduce the incidence of major postoperative complications. Additionally, we also explored the effect of HA380 hemoperfusion on coagulation, hemodynamics, and clinical outcomes. Upon completion of the study, we will elucidate the mechanism of hemoperfusion on SIRS. Further, this study helps to provide evidence for the application of HA380 in ATAAD patients undergoing $\mathrm{CPB}$ and $\mathrm{DHCA}$.

\section{Strengths and limitations of this study}

$>$ This is the first randomized controlled trial (RCT) to evalutae the effect of combined HA380 hemoperfusion and $\mathrm{CPB} / \mathrm{DHCA}$ versus conventional $\mathrm{CPB} / \mathrm{DHCA}$ in patients with ATAAD undergoing procedures involving the aortic arch. $>$ Interventions during $\mathrm{CPB} / \mathrm{DHCA}$ aims to decrease the levels of pro-inflammatory cytokines in the 
perioperative period and reduce severe postoperative complications.

$>$ This is a double-blind clinical trial. While it is not possible to blind the perfusionists from the patient's grouping, bias will be minimized by assigning other independent personnel for data collection at different clinical stages.

$\triangleright$ In the future, a large randomized controlled trial is needed, given the results of this single-center trial.

\section{Trial status}

The protocol was submitted to and approved by the Ethics Committee of the Second Affiliated Hospital of Nanjing Medical University (No.[2018] KY 118) on 20 December 2018. Patient recruitment started on 2 January 2019 and is expected to complete by 30 December 2020 (24 months). Following retrospective registration at ClinicalTrial.gov, the protocol was first released on 1 July 2019. The final protocol version is version 4, 29 February 2020.

\section{Abbreviations}

CPB: Cardiopulmonary bypass; SIRS: Significant systemic inflammatory response syndrome; ATAAD: Acute type A aortic dissection; DHCA: Deep hypothermic circulatory arrest; IL: Interleukin; TNF-a: Tumor necrosis factor-a; ICU: Intensive care unit; GCP: Good Clinical Practice; CONSORT: Consolidated Standards of Reporting Trials; CRF: Case report form; CTA: Computed tomography angiography; CRRT: Continuous renal replacement therapy; ECMO: Extracorporeal membrane oxygenation; ECG: Electrocardiogram; $\mathrm{SPO}_{2}$ : Blood oxygen saturation; HR: Heart rate; $\mathrm{rSO}_{2}$ : Regional cerebral oxygen saturation; ACT: Activated coagulation time; PO $3 \mathrm{M}$ : Postoperative 3 month; PO $6 \mathrm{M}$ : Postoperative 6 month; POD: Postoperative day; APTT: Activated partial thromboplastin time; PT: Prothrombin time; FIB: Fibrinogen; FDP: Fibrinogen degradation product; DDi: D-Dimer; ELISA: Enzyme-linked immunosorbent assay; MODS: Multiple organ dysfunction syndrome; MIF: Macrophage migration inhibitory factor; CRP: Creactive protein; SPIRIT: Standard Protocol Items: Recommendations for Interventional Trials; ANOVA: Analysis of variance; ANCOVA: Analysis of covariance; CARS: Compensatory anti-inflammatory response syndrome; CSAAKI: Cardiac surgery-associated acute kidney injury; RCT: Randomized controlled tria

\section{Acknowledgements}

Not applicable.

\section{Dissemination}

Study findings will be presented in meetings and published in medical journals.

\section{Authors' contributions}

Jing Yang designed the study, performed literature searching, drew the schematic, and drafted the manuscript. Dong Ji, Yue-Qian Zhu, and Hong-Yu Dai collected the blood sample and data. Yun Ren, Xu Sun, Yi Zhou, and ZhiYuan Chen recruited patients and contributed to the cardiopulmonary bypass. Xun Zhang advised on the study protocol and wrote the manuscript. Hao Yao and Qing-Guo Li conceived the idea and initiated the study. All authors have revised the study protocol and approved the final manuscript. All named authors adhere to the authorship guidelines of Trials. All authors have agreed to publication.

\section{Funding}

The study is supported by Postgraduate Research \& Practice Innovation Program of Jiangsu Province (SJCX190426) and National Natural Science Foundation of China (81670421). Funds have been used to support the conduct of the trial and the effort of investigators.

\section{Availability of data and materials}

Not applicable.

\section{Ethics approval and consent to participate}

The study was approved by the Ethics Committee of the Second Affiliated Hospital of Nanjing Medical University (No.[2018] KY 118) and registered at ClinicalTrials.gov (NCT 04007484). All patients admitted will be let know of the trial. If they wish to participate, a written informed consent needs to be provided before being recruited. On the consent form, participants will be asked if they agree to use their data should they choose to withdraw from the trial. Participants will also be asked for permission for the research team to share relevant data with people from the universities taking part in the research or from regulatory authorities, where relevant. This trial involves collecting biological specimens for storage.

\section{Consent for publication}

Not applicable.

\section{Competing interests}

The authors declare that they have no competing interests.

Received: 19 March 2020 Accepted: 1 November 2020

Published online: 23 November 2020

\section{References}

1. Go AS, Mozaffarian D, Roger VL, Benjamin EJ, Berry JD, Blaha MJ, Dai S, Ford ES, Fox CS, Franco S, et al. Heart disease and stroke statistics--2014 update: a report from the American Heart Association. Circulation. 2014;129(3):e28-e292.

2. Delannoy B, Guye ML, Slaiman DH, Lehot JJ, Cannesson M. Effect of cardiopulmonary bypass on activated partial thromboplastin time waveform analysis, serum procalcitonin and C-reactive protein concentrations. Crit Care. 2009;13(6):R180.

3. Hensel M, Volk T, Docke WD, Kern F, Tschirna D, Egerer K, Konertz W, Kox WJ. Hyperprocalcitonemia in patients with noninfectious SIRS and pulmonary dysfunction associated with cardiopulmonary bypass. Anesthesiology. 1998;89(1):93-104.

4. Salameh A, Dhein S. Strategies for pharmacological organoprotection during extracorporeal circulation targeting ischemia-reperfusion injury. Front Pharmacol. 2015;6:296

5. Sugita J, Fujiu K. Systemic inflammatory stress response during cardiac surgery. Int Heart J. 2018;59(3):457-9.

6. Baumann A, Buchwald D, Annecke T, Hellmich M, Zahn PK, Hohn A. RECCAS - REmoval of cytokines during CArdiac surgery: study protocol for a randomised controlled trial. Trials. 2016;17(1):137.

7. McGuinness J, Bouchier-Hayes D, Redmond JM. Understanding the inflammatory response to cardiac surgery. Surgeon. 2008;6(3):162-71.

8. Squiccimarro E, Labriola C, Malvindi PG, Margari V, Guida P, Visicchio G, Kounakis G, Favale A, Dambruoso P, Mastrototaro G, et al. Prevalence and clinical impact of systemic inflammatory reaction after cardiac surgery. J Cardiothorac Vasc Anesth. 2019;33(6):1682-90.

9. Allan CK, Newburger JW, McGrath E, Elder J, Psoinos C, Laussen PC, del Nido PJ, Wypij D, McGowan FX Jr. The relationship between inflammatory activation and clinical outcome after infant cardiopulmonary bypass. Anesth Analg. 2010;111(5):1244-51.

10. Whitlock RP, Devereaux PJ, Teoh KH, Lamy A, Vincent J, Pogue J, Paparella D, Sessler DI, Karthikeyan G, Villar JC, et al. Methylprednisolone in patients undergoing cardiopulmonary bypass (SIRS): a randomised, double-blind, placebo-controlled trial. Lancet. 2015:386(10000):1243-53.

11. Basu R, Pathak S, Goyal J, Chaudhry R, Goel RB, Barwal A. Use of a novel hemoadsorption device for cytokine removal as adjuvant therapy in a patient with septic shock with multi-organ dysfunction: a case study. Indian J Crit Care Med. 2014;18(12):822-4.

12. Bernardi MH, Rinoesl H, Dragosits K, Ristl R, Hoffelner F, Opfermann P, Lamm C, Preissing F, Wiedemann D, Hiesmayr MJ, et al. Effect of hemoadsorption during cardiopulmonary bypass surgery - a blinded, randomized, controlled pilot study using a novel adsorbent. Crit Care. 2016;20:96.

13. Futier E, Biais M, Godet T, Bernard L, Rolhion C, Bourdier J, Morand D, Pereira B, Jaber S, Committee FTM. Fluid loading in abdominal surgery saline versus hydroxyethyl starch (FLASH trial): study protocol for a randomized controlled trial. Trials. 2015;16:582. 
14. Callum J, Farkouh ME, Scales DC, Heddle NM, Crowther M, Rao V, Hucke HP, Carroll J, Grewal D, Brar S, et al. Effect of fibrinogen concentrate vs cryoprecipitate on blood component transfusion after cardiac surgery: the FIBRES randomized clinical trial. JAMA. 2019;322(20):1-11.

15. Poli EC, Alberio L, Bauer-Doerries A, Marcucci C, Roumy A, Kirsch M, De Stefano E, Liaudet L, Schneider AG. Cytokine clearance with CytoSorb(R) during cardiac surgery: a pilot randomized controlled trial. Crit Care. 2019;23(1):108.

16. Pomare Montin D, Ankawi G, Lorenzin A, Neri M, Caprara C, Ronco C. Biocompatibility and cytotoxic evaluation of new sorbent cartridges for blood Hemoperfusion. Blood Purif. 2018;46(3):187-95.

17. Khwaja A. KDIGO clinical practice guidelines for acute kidney injury. Nephron Clin Pract. 2012;120(4):C179-84.

18. Hogue CW Jr, Sundt T 3rd, Barzilai B, Schecthman KB, Davila-Roman VG. Cardiac and neurologic complications identify risks for mortality for both men and women undergoing coronary artery bypass graft surgery. Anesthesiology. 2001;95(5):1074-8.

19. Hessel EA 2nd. Abdominal organ injury after cardiac surgery. Semin Cardiothorac Vasc Anesth. 2004;8(3):243-63.

20. Raman JS, Kochi K, Morimatsu H, Buxton B, Bellomo R. Severe ischemic early liver injury after cardiac surgery. Ann Thorac Surg. 2002;74(5):1601-6.

21. Subramaniam B, Shankar P, Shaefi S, Mueller A, O'Gara B, Banner-Goodspeed V, Gallagher J, Gasangwa D, Patxot M, Packiasabapathy S, et al. Effect of intravenous acetaminophen vs placebo combined with propofol or dexmedetomidine on postoperative delirium among older patients following cardiac surgery: the DEXACET randomized clinical trial. JAMA. 2019;321(7):686-96.

22. Almdahl SM, Rynning SE, Veel T, Halvorsen P, Molstad P. Postoperatively increased serum alanine aminotransferase level is closely associated with mortality after cardiac surgery. Thorac Cardiovasc Surg. 2015;63(1):67-72.

23. Thygesen K, Alpert JS, Jaffe AS, Chaitman BR, Bax JJ, Morrow DA, White HD. Executive Group on behalf of the Joint European Society of Cardiology /American College of Cardiology /American Heart Association Norld Heart Federation Task Force for the Universal Definition of Myocardial I: Fourth Universal Definition of Myocardial Infarction (2018). Circulation. 2018;138(20):e618-51.

24. Bonavia A, Groff A, Karamchandani K, Singbartl K. Clinical utility of extracorporeal cytokine hemoadsorption therapy: a literature review. Blood Purif. 2018;46(4):337-49.

25. Trager K, Fritzler D, Fischer G, Schroder J, Skrabal C, Liebold A, Reinelt H. Treatment of post-cardiopulmonary bypass SIRS by hemoadsorption: a case series. Int J Artif Organs. 2016;39(3):141-6.

26. Dinarello CA. Historical insights into cytokines. Eur J Immunol. 2007;37(Suppl 1):S34-45.

27. Nebelsiek T, Beiras-Fernandez A, Kilger E, Mohnle P, Weis F. Routine use of corticosteroids to prevent inflammation response in cardiac surgery. Recent Pat Cardiovasc Drug Discov. 2012;7(3):170-4.

28. Andersson U, Tracey KJ. Reflex principles of immunological homeostasis. Annu Rev Immunol. 2012;30:313-35.

29. Gerlach H. Agents to reduce cytokine storm. F1000Res. 2016;5:2909.

30. Datzmann T, Trager K. Extracorporeal membrane oxygenation and cytokine adsorption. J Thorac Dis. 2018;10(Suppl 5):S653-60.

31. Matsuda K, Moriguchi T, Harii N, Goto J. Comparison of efficacy between continuous hemodiafiltration with a PMMA membrane hemofilter and a PAN membrane hemofilter in the treatment of a patient with septic acute renal failure. Transfus Apher Sci. 2009:40(1):49-53.

32. Hosgood SA, Moore T, Kleverlaan T, Adams T, Nicholson ML. Haemoadsorption reduces the inflammatory response and improves blood flow during ex vivo renal perfusion in an experimental model. J Transl Med. 2017;15(1):216

33. Franke A, Lante W, Fackeldey V, Becker HP, Thode C, Kuhlmann WD, Markewitz A. Proinflammatory and antiinflammatory cytokines after cardiac operation: different cellular sources at different times. Ann Thorac Surg. 2002;74(2):363-70 discussion 370-361.

34. Allen ML, Hoschtitzky JA, Peters MJ, Elliott M, Goldman A, James I, Klein NJ. Interleukin-10 and its role in clinical immunoparalysis following pediatric cardiac surgery. Crit Care Med. 2006;34(10):2658-65.

35. Calandra T, Roger T. Macrophage migration inhibitory factor: a regulator of innate immunity. Nat Rev Immunol. 2003;3(10):791-800.

36. Leyton-Jaimes MF, Kahn J, Israelson A. Macrophage migration inhibitory factor: a multifaceted cytokine implicated in multiple neurological diseases. Exp Neurol. 2018;301(Pt B):83-91.

37. Stoppe C, Averdunk L, Goetzenich A, Soppert J, Marlier A, Kraemer S, Vieten J, Coburn M, Kowark A, Kim BS, et al. The protective role of macrophage migration inhibitory factor in acute kidney injury after cardiac surgery. Sci Transl Med. 2018;10(441):eaan4886.

38. Vercaemst L. Hemolysis in cardiac surgery patients undergoing cardiopulmonary bypass: a review in search of a treatment algorithm. J Extra Corpor Technol. 2008;40(4):257-67.

39. Murphy GS, Whitlock RP, Gutsche JT, Augoustides JG. Steroids for adult cardiac surgery with cardiopulmonary bypass: update on dose and key randomized trials. J Cardiothorac Vasc Anesth. 2013;27(5):1053-9.

\section{Publisher's Note}

Springer Nature remains neutral with regard to jurisdictional claims in published maps and institutional affiliations.

\section{Ready to submit your research? Choose BMC and benefit from:}

- fast, convenient online submission

- thorough peer review by experienced researchers in your field

- rapid publication on acceptance

- support for research data, including large and complex data types

- gold Open Access which fosters wider collaboration and increased citations

- maximum visibility for your research: over $100 \mathrm{M}$ website views per year

At BMC, research is always in progress.

Learn more biomedcentral.com/submissions 